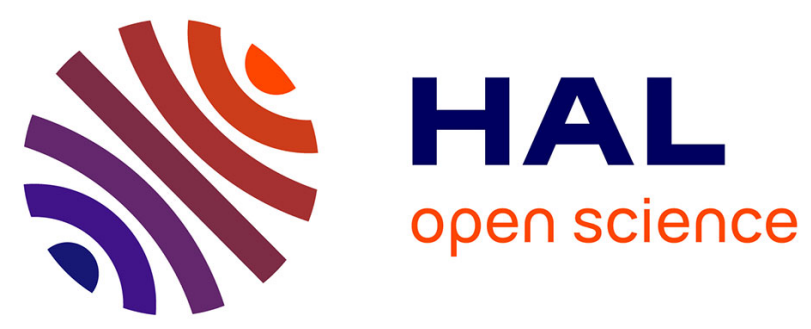

\title{
Characteristics of the phase noise correlation of injection locked lasers for RF signal generation and transmission
}

Fabien Kéfélian, Renaud Gabet, Philippe Gallion

\section{To cite this version:}

Fabien Kéfélian, Renaud Gabet, Philippe Gallion. Characteristics of the phase noise correlation of injection locked lasers for RF signal generation and transmission. Optical and Quantum Electronics, 2006, 38, pp.467-478. 10.1007/s11082-006-0043-4 . hal-01075173

\section{HAL Id: hal-01075173 \\ https://hal.science/hal-01075173}

Submitted on 8 Jan 2015

HAL is a multi-disciplinary open access archive for the deposit and dissemination of scientific research documents, whether they are published or not. The documents may come from teaching and research institutions in France or abroad, or from public or private research centers.
L'archive ouverte pluridisciplinaire $\mathbf{H A L}$, est destinée au dépôt et à la diffusion de documents scientifiques de niveau recherche, publiés ou non, émanant des établissements d'enseignement et de recherche français ou étrangers, des laboratoires publics ou privés. 


\title{
Characteristics of the phase noise correlation of injection locked lasers for RF signal generation and transmission
}

\author{
F. KÉFÉLIA N*, R. GABET A N P P. GALLION \\ École Nationale Supérieure des Télécommunications (GET / Télécom Paris and CNRS UMR 5141 \\ LTCI) 46 rue Barrault, 75634 Paris cedex 13, France \\ (*author for correspondence: E-mail: kefelian@enst.fr)
}

Received 24 June 2005; accepted 26 January 2006

\begin{abstract}
Theoretical and experimental results on phase noise characteristics of an optical radio-frequency generation and transmission system using heterodyne photo-detection and side band injection locking is reported. Optical phase noise correlation of the lasers is achieved by noise transfer from a modulated master laser. Our investigation takes into account phase noise enhancement in optical comb generation, injection dynamic, path delay, and fiber dispersion. Theoretical expressions are derived from an adiabatic approximation and compared with experimental measures.
\end{abstract}

Key words: injection locking, optical correlation, phase noise, radio-over-fiber

\section{Introduction}

Radio-over-Fiber (RoF) technology is based on the deployment and the integration of the optical fiber in wireless networks, providing microwave signals distribution by means of optical links and components. This technology shifts the system complexity from the remote base stations to a single central station, reducing the cost of each base station. It can increase the networks capacity using smaller cell dimension with dynamic frequency allocation. The light source in the central station is modulated by the radio signal carrying the data and transmitted over fiber toward base stations. A photodiode receiver is located at the base station for down-conversion before wireless diffusion.

Mobile Broadband Systems (MBS) provides radio coverage restricted to a small area and is a solution to demand for mobility. It works at the picocell level and at data rate up to $155 \mathrm{Mb} / \mathrm{s}$. The radio transmission operation at $60 \mathrm{GHz}$ reduces dramatically the line-of-sight coverage because of $\mathrm{O}_{2}$ molecular absorption and is consequently a way to reduce the cell size and multiuser interference (Schmuck et al. 1994).

The MBS can be combined with RoF technology, but the carrier frequency range is beyond the possibility of direct modulation of the laser 
source. One of the solutions is to directly modulate the laser with an intermediate frequency carrying the data and to use optical generation of microwave signals to provide frequency conversion at the base station stage. Optical generation of the microwave carrier is based on heterodyne photo-detection. A second laser, unmodulated, is inserted in the central station with a frequency difference equal to the target microwave frequency.

This method assures a maximum intensity modulation depth but it requires sources with phase correlation to cancel out the large intrinsic optical phase noise effect. Without correlation, the beat note exhibits strong phase noise and slow thermal mean frequency drift. Our experimental setting uses the FM-sideband optical injection locking method to correlate both slave lasers (Goldberg et al. 1983; Braun and Grosskopf 1998). This method assures a perfect control of the generated microwave frequency, an efficient optical phase noise rejection and a direct base-band data phase shift modulation ability. In some other experiments, the FM sidebands comb has been replaced by a multi-tone photonic oscillator (Sayyah and Yap 2003), or a mode-locked laser frequency comb (Logan 1994) in order to extend the frequency span.

Using efficient modulation format, like M-PSK and M-QAM, narrower channel spacing can be obtained, but it requires high-spectral purity carrier. Consequently, it is essential to investigate all sources of phase noise and of correlation loss from the optical sources in central station to the base station remote antenna. Three sources of correlation loss are theoretically and experimentally investigated: IM/FM modulation of the master laser, phase noise transfer by injection locking of slave lasers, and finally the transmission of the two optical frequencies on the optical fiber.

\section{Correlation transfer in the sideband injection locking method}

The phase of the beat signal of two lasers is given by the formula:

$$
\phi_{\mathrm{RF}}(t)=\phi_{1}(t)-\phi_{2}(t),
$$

where $\phi_{1}$ and $\phi_{2}$ are the phase of the optical field on the photo-detector surface. The mean square of the RF phase noise is:

$$
\left\langle\phi_{\mathrm{RF}}^{2}\right\rangle=\left\langle\phi_{1}^{2}\right\rangle+\left\langle\phi_{1}^{2}\right\rangle-2\left\langle\phi_{1} \phi_{2}\right\rangle .
$$

Consequently, to avoid the transfer of strong optical phase noise on the RF carrier, optical phases must be strongly correlated.

To characterize this correlation, we use the cross power spectral density function (XPD) formally defined as: 
PHASE NOISE CORRELATION OF INJECTION LOCKED LASERS

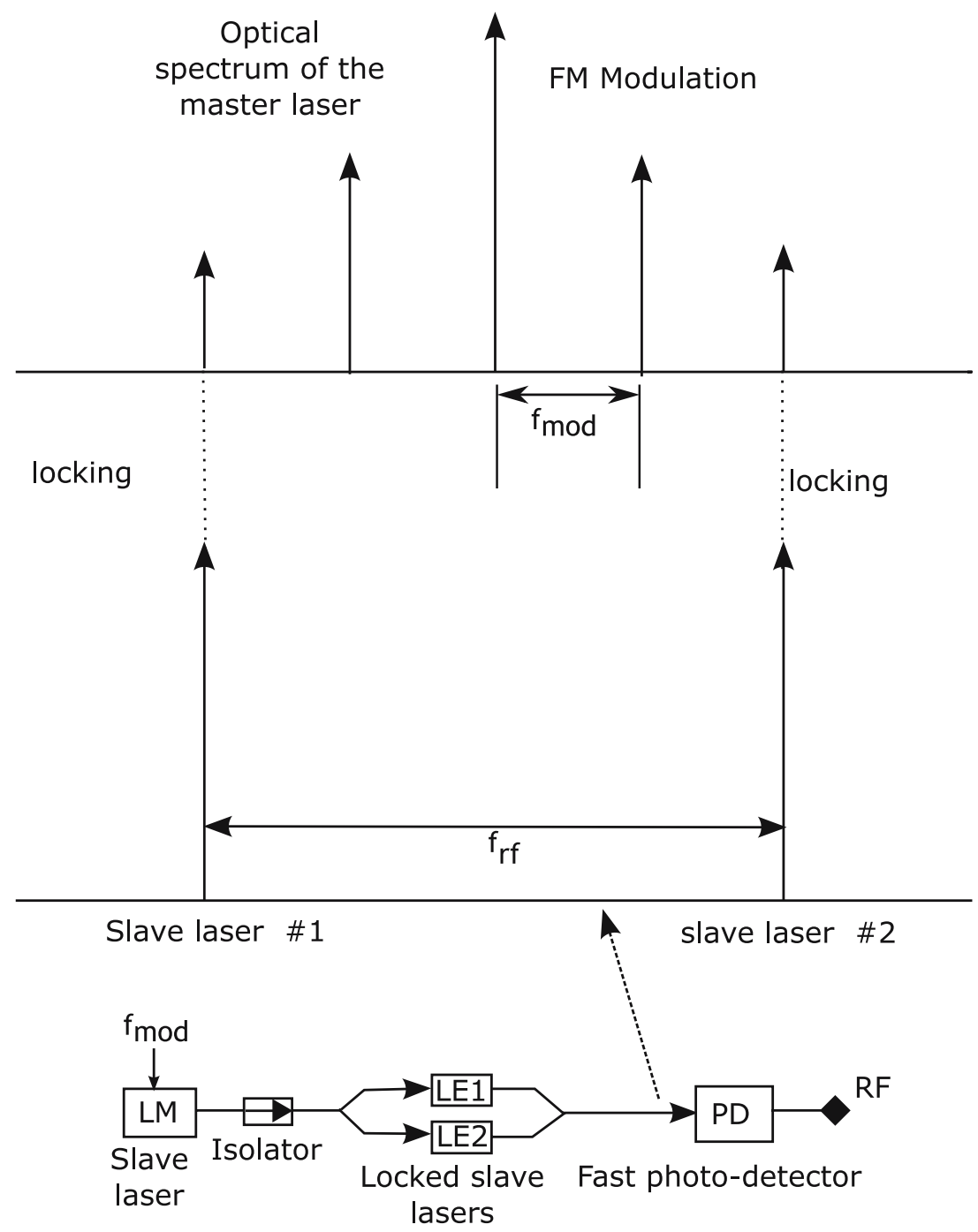

Fig. 1. Side band injection locking method.

$$
S_{\phi_{1} \phi_{2}}(f) \delta\left(f-f^{\prime}\right)=\left\langle\phi_{1}(f) \phi_{2}^{*}\left(f^{\prime}\right)\right\rangle
$$

in addition with the usual power spectral density (PSD)

$$
S_{\phi_{1}}(f) \delta\left(f-f^{\prime}\right)=\left\langle\phi_{1}(f) \phi_{1}^{*}\left(f^{\prime}\right)\right\rangle .
$$




\subsection{FM-SIDEBAND INJECTION METHOD}

The FM-sideband injection locking is a very efficient method to correlate two or more slave lasers with different optical frequencies. Using the IM/FM coupling in the direct modulation of a semiconductor laser, we obtain a phase correlated optical comb with a usual RF generator with frequency inside the modulation bandwidth of the laser. Each tooth of the comb can become a master source for injection locking of a slave laser. If two lasers are locked on different teeth, their frequency difference is an exact multiple of the sub-harmonic frequency.

\subsection{CORRELATION OF THE OPTICAL COMB}

When optical injection locking is used to correlate lasers of different frequencies, the quality of the correlation is originally determined by the quality of the correlation of the selected teeth of the comb.

When the optical comb is the result of a FM modulation induced by current modulation, the phase of the optical side-band corresponding to the harmonic $\# n$ is given by Bessel expansion:

$$
\phi_{n}(t)=\phi_{\mathrm{opt}}(t)+n \phi_{\mathrm{osc}}(f),
$$

where $\phi_{n}$ is the phase of the optical side band $\# n, \phi_{\text {opt }}$ is the phase of the laser without modulation, and $\phi_{\text {osc }}$ is the phase of the modulating radiowave. The phase noise PSD of the side band \#n is:

$$
S_{\phi_{n}}(f)=S_{\phi_{\mathrm{opt}}}(f)+n^{2} S_{\phi_{\mathrm{osc}}}(f)
$$

and the correlation between $\phi_{n}$ and $\phi_{n^{\prime}}$ is spectrally given by:

$$
S_{\phi_{n} \phi_{n^{\prime}}}(f)=S_{\phi_{\mathrm{opt}}}(f)+n n^{\prime} S_{\phi_{\mathrm{osc}}}(f) .
$$

Both side-modes are not fully correlated:

$$
\left|S_{\phi_{n} \phi_{n^{\prime}}}(f)\right|<\sqrt{S_{\phi_{n}}(f) S_{\phi_{n^{\prime}}}(f) .}
$$

Consequently, even when the linewidth of the RF modulation oscillator is negligible compared to the laser linewidth, the multiplied RF phase noise on each optical tooth has to be taken into account, because it induces initial uncorrelation in the master optical comb. 


\subsection{THE NOISE TRANSFER AND REJECTION IN INJECTION LOCKING}

\subsubsection{Adiabatic calculation of the phase noise of the locked laser}

The goal of this section is to establish a simplified expression of the phase noise of an injection locked slave laser using the adiabatic suppression of the carrier density in the phase equation. This approximation is valid for the usual carrier offset frequencies of the RF phase noise spectrum usually spanning from $100 \mathrm{~Hz}$ to $10 \mathrm{MHz}$, far below the relaxation frequency of the free slave laser. In the adiabatic approximation, the gain saturation is instantaneous, and the carrier shot noise is ignored.

We start the calculation using the optical output field envelope $A(t)$ equation with injection (Lang 1982):

$$
\begin{aligned}
\frac{\mathrm{d} A^{+}}{\mathrm{d} t}(t)= & -j\left(\omega_{\mathrm{s}}-\omega_{i}\right) A^{+}(t)+A^{+}(t) \frac{\left(1+j \alpha_{\mathrm{H}}\right)}{2} v_{\mathrm{g}} g_{N} \Delta N(t) \\
& +f_{\mathrm{R}} t_{2} A^{\mathrm{in}}(t)+F_{\mathrm{A}}(t),
\end{aligned}
$$

where $\omega_{\mathrm{s}} / 2 \pi$ is the optical frequency of the slave laser without injection, $\omega_{i} / 2 \pi$ the master laser optical frequency, $A^{\text {in }}(t)$ the field envelope of the master laser at the input of the slave laser, $t_{2}$ the amplitude transmission of the injection facet, $\alpha_{\mathrm{H}}$ the Henry factor, $v_{\mathrm{g}}$ the group velocity, $\frac{\partial g}{\partial N}=g_{N}$ and $g$ the modal gain of the slave laser, and $\Delta N$ is the carrier density deviation from the free stationary solution induced by injection. $F_{\mathrm{A}}$ is the Langevin force taking into account the spontaneous emission. The $f_{\mathrm{R}}$ is a coupling factor with frequency dimension, for injection through the output facet of a Fabry-Perot laser, $f_{\mathrm{R}}=v_{\mathrm{g}} / 2 l r_{2}$, where $l$ is the cavity length and $r_{2}$ the amplitude reflectivity of the injection facet, for injection through the output facet of a DFB laser, $f_{\mathrm{R}}$ is proportional to the Favre factor (Favre 1987) for optical feedback in DFB (Campuzano et al. 2001).

Multiplying (9) by $2 A^{+*}(t)$ and considering real and imaginary part, we obtain the phase equation and the output power equation:

$$
\begin{aligned}
& \frac{\mathrm{d} I}{\mathrm{~d} t}(t)=v_{\mathrm{g}} g_{N} \Delta N(t) I(t)+2 f_{\mathrm{R}} T_{2} \sqrt{I(t) I_{\mathrm{in}}(t)} \cos \left(\phi_{\mathrm{in}}(t)-\phi_{s}(t)\right)+F_{I}(t), \quad(10) \\
& \frac{\mathrm{d} \phi_{s}}{\mathrm{~d} t}(t)=\left(\omega_{\mathrm{s}}-\omega_{i}\right)+\frac{\alpha_{\mathrm{H}}}{2} v_{\mathrm{g}} g_{N} \Delta N(t)+f_{\mathrm{R}} T_{2} \sqrt{\frac{I_{\text {in }}}{I(t)}} \sin \left(\phi_{\mathrm{in}}(t)-\phi_{s}(t)\right)+F_{\phi}(t) .
\end{aligned}
$$

Considering the adiabatic approximations, the small signal regime and neglecting the RIN against the phase noise:

$$
\frac{\mathrm{d} \phi_{s}}{\mathrm{~d} t}(t)=\delta \theta(t) \delta \omega_{\max } \cos \left(\theta_{0}-\tan ^{-1}\left(\alpha_{\text {eff }}\right)\right)+F_{\phi}(t)+\alpha_{\text {eff }} \frac{F_{I}(t)}{2 I_{0}},
$$


where $\delta \omega_{\max }$ is the half-angular frequency locking bandwidth and $\delta \theta$ is the lasers phases difference noise.

$$
\delta \omega_{\max }=f_{\mathrm{R}} \eta \sqrt{1+\alpha_{\mathrm{H}}^{2}}
$$

where $\eta=\sqrt{\frac{I_{\text {in } 0} T_{2}}{I_{0} / T_{2}}}, I_{\text {in } 0}$ is the mean master power at the input of the injection facet, $I_{0}$ the mean output power of the slave laser and $T_{2}$ is the intensity transmission factor. $F_{I}$ and $F_{\phi}$ are the Langevin forces associated to phase and power fluctuations induced by spontaneous emission, with the diffusion coefficients(Henry 1983):

$$
2 D_{I I}=2 R_{\mathrm{sp}} \frac{I_{0}^{2}}{p_{0}}, \quad 2 D_{\phi \phi}=\frac{R_{\mathrm{sp}}}{2 p_{0}}
$$

where $p_{0}$ are the total number of photons inside the cavity. $R_{\mathrm{sp}}$ is the spontaneous rate of emission. For a DFB laser, or for a laser with gain compression, $\alpha_{\mathrm{H}}$ must be replaced by a modified coefficient $\alpha_{\text {eff }}$ taking into account $\Delta I$ and the modification of the longitudinal mode.

Taking the Fourier transform of (12):

$$
\phi_{s}(f)=\frac{2 \pi k_{c}^{\prime} \phi_{\mathrm{in}}(f)+F_{\phi}(f)+\alpha_{\mathrm{eff}} \frac{F_{I}(f)}{2 I_{0}}}{j 2 \pi f+2 \pi k_{c}^{\prime}},
$$

where

$$
k_{c}^{\prime}=\frac{\delta \omega_{\max }}{2 \pi} \cos \left(\theta_{0}-\tan ^{-1}\left(\alpha_{\text {eff }}\right)\right),
$$

we obtain:

$$
S_{\phi_{s}}(f)=\underbrace{\frac{k_{c}^{\prime 2}}{k_{c}^{\prime 2}+f^{2}} S_{\phi_{\mathrm{in}}}(f)}_{(a)}+\underbrace{\frac{f^{2}}{k_{c}^{\prime 2}+f^{2}} S_{\phi_{l}}(f)}_{(b)}
$$

with $S_{\phi_{l}}(f)$ the free slave phase noise:

$$
S_{\phi_{l}}(f)=\frac{\Delta v}{2 \pi f^{2}},
$$

where $\Delta v=\left(1+\alpha^{2}\right) \frac{R_{\mathrm{sp}}}{4 \pi p_{0}}$ is the linewidth without injection.

The locked laser phase noise PSD (17) is the sum of two contributions as follows: 
- (a) comes from the phase noise transmitted by the master laser, as with a first-order phase locked loop of locking frequency $k_{c}^{\prime}$,

- (b) comes from the remaining (unlocked) phase noise of the free slave laser.

The XPD between slave and master phase noise is:

$$
S_{\phi_{\mathrm{s}} \phi_{\mathrm{m}}}(f)=\frac{k_{c}^{\prime} S_{\phi_{\mathrm{m}}}(f)}{j f+k_{c}^{\prime}}
$$

master and slave are not fully spectrally correlated: $\left|S_{\phi_{\mathrm{m}} \phi_{\mathrm{s}}}(f)\right|<$ $\sqrt{S_{\phi_{\mathrm{m}}}(f) S_{\phi_{\mathrm{s}}}(f)}$.

\subsection{DELAY EFFECT ON CORRELATION}

During the transmission of the "virtual" RF signal as a correlated optical wave doublet, phase equality have to be carefully managed. The main delay takes place when both optical waves are combined after a different propagation path, but the dispersion is also a source of delay between the two optical frequencies in the same fiber.

When the phase $\phi_{1}$ is delayed by a duration $\Delta \tau$ compared with $\phi_{2}$, the XPD between both phase is modified the following way:

$$
S_{\phi_{1}(\Delta \tau) \phi_{2}}=S_{\phi_{1} \phi_{2}} \mathrm{e}^{-j 2 \pi f \Delta \tau}
$$

consequently, the delay induces a rotation of the XPD, i.e., a modification of the mean phase shift between spectral components of the phases. If the XPD becomes a real negative number, the Fourier components $\phi_{1}(f)$ and $\phi_{2}(f)$ become anti-correlated and will be added after heterodyne detection.

True and dispersion delays can be easily calculated using the arms length of the couplers (in the configuration of our system, injection, and emission are done through the same facet), the dispersion coefficient, the transmission fiber length and the frequency difference. Since the geometric position of each slave lasers can be adjusted, a true delay may be introduced in the faster frequency arm to compensate the dispersion delay:

$$
\Delta \tau=L\left(\beta^{\prime}\left(v_{1}\right)-\beta^{\prime}\left(v_{2}\right)\right) \approx-\frac{D L \lambda_{0}^{2} f_{\mathrm{RF}}}{c},
$$

where $\beta$ is the propagation constant in the fiber, $D$ is the chromatic dispersion parameter. This compensation is strictly valid only for the two single optical frequencies $v_{1}$ and $v_{2}$, i.e., for the beat frequency $\left|v_{1}-v_{2}\right|=f_{\mathrm{RF}}$. The compensation is second-order valid for other components of the lasers optical spectra separated by $f_{\mathrm{RF}}$, the third-order effect taking into account the 
slop dispersion difference between $v_{1}$ and $v_{1}$ for $f_{\mathrm{RF}}<100 \mathrm{GHz}$ has negligible effect on the RF phase noise. For beating between other spectral components of the optical lineshapes, the residual effect on the RF spectrum is equivalent to the phase-to-intensity noise conversion induced by a dispersion media on one optical lineshape (Yamamoto et al. 1990) and its contribution is negligible for moderate length standard fiber and $\mathrm{MHz}-$ linewidth lasers $(<-160 \mathrm{dBc} / \mathrm{Hz}$ for $10-\mathrm{km}$ fiber and $1-\mathrm{MHz}$ linewidth).

\subsection{CORRELATION OF THE TWO INJECTION LOCKED LASERS}

Now we consider two lasers with linewidth $\Delta v_{1}$ and $\Delta v_{1}$, injection locked on side bands $\# n$ and $\# n^{\prime}$ with parameters $k_{1}^{\prime}$ et $k_{2}^{\prime}$. Using Equation (19), the XPD of both phases is given by:

$$
S_{\phi_{1} \phi_{2}}(f)=\frac{k_{c 1}^{\prime} k_{c 2}^{\prime} S_{\phi_{n} \phi_{n^{\prime}}}(f)}{f^{2}+j f\left(k_{c 1}^{\prime}-k_{c 2}^{\prime}\right)+k_{c 1}^{\prime} k_{c 2}^{\prime}} .
$$

If $k_{c 1}^{\prime}=k_{c 2}^{\prime}$ :

$$
S_{\phi_{1} \phi_{2}}(f)=\frac{k_{c}^{\prime 2} S_{\phi_{n} \phi_{n^{\prime}}}(f)}{f^{2}+k_{c}^{\prime 2}} .
$$

The expression of each laser phase noise PSD is given by Equation (17):

$$
\begin{aligned}
& S_{\phi_{1}}(f)=\frac{k_{c}^{\prime 2}}{k_{c}^{\prime 2}+f^{2}}\left(S_{\phi_{\mathrm{M}}}+n^{2} S_{\phi_{\mathrm{osc}}}\right)+\frac{f^{2}}{k_{c}^{\prime 2}+f^{2}} S_{\phi_{1 l}}(f), \\
& S_{\phi_{2}}(f)=\frac{k_{c}^{\prime 2}}{k_{c}^{\prime 2}+f^{2}}\left(S_{\phi_{\mathrm{M}}}+n^{\prime 2} S_{\phi_{\mathrm{osc}}}\right)+\frac{f^{2}}{k_{c}^{\prime 2}+f^{2}} S_{\phi_{2 l}}(f) .
\end{aligned}
$$

2.6. PHASE NOISE OF THE BEAT NOTE

The PSD of the beat note is given by:

$$
S_{\phi_{\mathrm{RF}}}(f)=S_{\phi_{1}}(f)+S_{\phi_{2}}(f)-2 \Re e\left\{S_{\phi_{1} \phi_{2}}(f)\right\} .
$$

We see now that the spectral rotation induced by the delay reduces the effect of the correlation: 


$$
\begin{aligned}
\Re e\left\{S_{\phi_{1}(\Delta \tau) \phi_{2}}(f)\right\} & =\frac{k_{c}^{\prime 2} S_{\phi_{n} \phi_{n^{\prime}}}(f)}{f^{2}+{k_{c}^{\prime}}^{2}} \cos (2 \pi f \Delta \tau) \\
& =\frac{{k_{c}^{\prime}}^{2}}{f^{2}+{k_{c}^{\prime}}^{2}} \cos (2 \pi f \Delta \tau)\left(S_{\phi_{\mathrm{M}}}(f)+n n^{\prime} S_{\phi_{\mathrm{M}}}(f)\right) .
\end{aligned}
$$

Using the expression of each laser phase noise PSD and the XPD, we obtain:

$$
\begin{aligned}
S_{\phi_{\mathrm{RF}}}(f)= & \frac{k_{c}^{\prime 2}}{{k_{c}^{\prime}}^{2}+f^{2}}\left[4 S_{\phi_{\mathrm{M}}}(f) \sin (\pi f \Delta \tau)+\left|n-n^{\prime} \mathrm{e}^{2 \pi f \Delta \tau}\right|^{2} S_{\phi_{\mathrm{osc}}}(f)\right] \\
& +\frac{f^{2}}{{k_{c}^{\prime}}^{2}+f^{2}}\left[S_{\phi_{1 l}}(f)+S_{\phi_{2 l}}(f)\right],
\end{aligned}
$$

if $\Delta \tau=0$,

$$
S_{\phi_{\mathrm{RF}}}(f)=\frac{k_{c}^{\prime 2}}{k_{c}^{\prime 2}+f^{2}}\left[\left(n-n^{\prime}\right)^{2} S_{\phi_{\mathrm{osc}}}(f)\right]+\frac{f^{2}}{k_{c}^{\prime 2}+f^{2}}\left[S_{\phi_{1 l}}(f)+S_{\phi_{2 l}}(f)\right] .
$$

\subsection{OTHER NOISE SOURCES}

In Equation (12), we have neglected the intensity noise of the master and slave lasers. The intensity noise has usually negligible influence on the optical spectrum of a diode laser dominated by the phase noise. But for RF beat lasers generation, the RIN can have an impact on the amplitude noise of the RF beat note and cannot be neglected in presence of a strong $1 / f$ component in the RIN spectrum (Bibey et al. 1999).

We have also neglected the shot noise of the carriers, but the pump source of the slave laser can have additional noises. Those contributions can be included in Equation (12) through a current fluctuation Langevin force with a correlation product determined by the power spectrum of the DC source (Agrawal and Roy 1988), and using the modulation transfer function of the injection locked laser (Lidoyne et al. 1991), with thermal effect for low frequencies (Petermann 1988).

\section{Numerical analysis}

We have summarized in Fig. 2 the different phase noise penalties induced by various parameters previously studied: slave laser linewidth, locking bandwidth, delay, and master linewidth. In all cases, the spectrum exhibits a noise floor below the injection locking frequency corresponding to the 

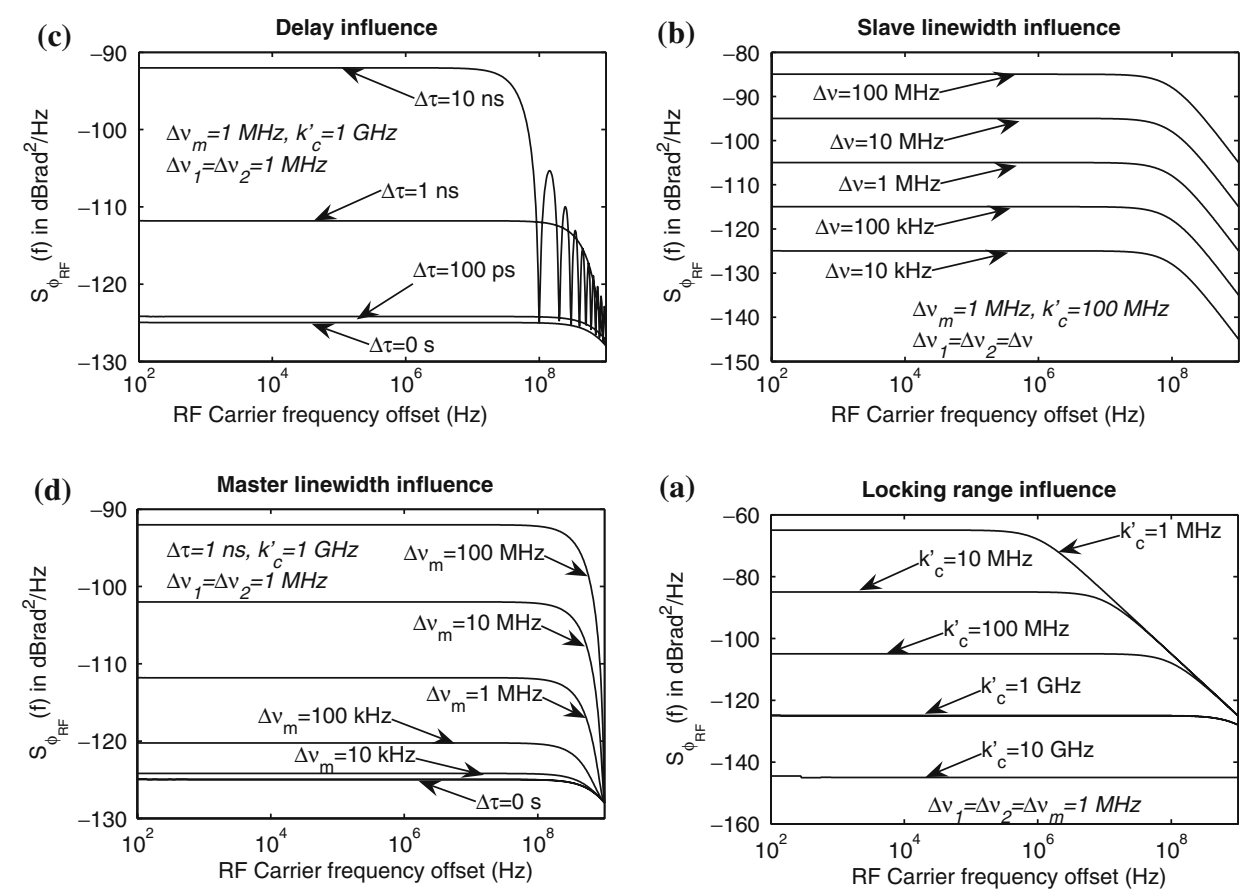

Fig. 2. RF phase power spectral density.

phase/frequency noise conversion, and a $1 / f^{2}$ decrease above, corresponding to the free laser phase noise. Consequently, we discuss hereafter the level of the noise floor.

- The locking range influence on the noise floor showed in Fig. 2(a) is characterized by a penalty of $-20 \mathrm{~dB}$ for each decade of $k_{c}^{\prime}$.

- The slave linewidth influence on the noise floor showed in Fig. 2(b) is characterized by a penalty of $-10 \mathrm{~dB}$ for each decade of $\Delta v$.

- The parameter to consider for the delay influence shown by Fig. 2(c) and (d) is the product $\Delta v_{\mathrm{m}} \Delta \tau^{2}$, consequently a narrow spectrum master laser induces a less delay sensitive system.

\section{Experimental results}

Experimental measurements were achieved with three distributed feedback multi-quantum wells lasers provided by Alcatel Research and Innovation at $1548 \mathrm{~nm}, \Delta v \approx 0.8 \mathrm{MHz}, \alpha_{\mathrm{H}} \approx 3, I_{\mathrm{th}} \approx 10 \mathrm{~mA}$ at $25^{\circ} \mathrm{C}, P_{\max } \approx 40 \mathrm{~mW}$. The 3-GHz sub-harmonic source is a Rohde \& Schwartz SMP02/22. The two slave lasers are locked on harmonics \#+2 and \#-3, consequently the 
frequency of the beat note is $15 \mathrm{GHz}$, but we are able to obtain RF signal up to $100 \mathrm{GHz}$ with appropriate detector. The injection is achieved with zero detuning corresponding to a maximum of $k_{c}^{\prime}$ for a given injection rate. The locking bandwidth is controlled with an optical attenuator and set to be equal for both lasers.

We have measured the phase noise spectrum $(L(f))$ for four different locking bandwidths as reported in Fig. 3. Due to the phase noise of the analyzer, results are only clearly visible in the frequency offset range $(10 \mathrm{kHz}, 1 \mathrm{MHz})$.

The uniform $15 \mathrm{~dB}$ penalty corresponds to the multiplication effect on the sub-harmonic oscillator phase noise, i.e. the correlation of the master comb. We have represented in mixed line the theoretical noise floor corresponding to the locking bandwidth 100,45 , and $25 \mathrm{MHz}$. The measures are in good agreement with the formulas previously derived. We can notice, with the $25-\mathrm{MHz}$ locking bandwidth, the apparition of a $1 / f$ component for frequencies below $5 \mathrm{kHz}$, probably corresponding to the $1 / f$ component of the frequency noise of the slave lasers.

Measurements achieved with a 2-GHz locking bandwidth at frequencies offset above $50 \mathrm{MHz}$ revealed a noise floor at $-110 \mathrm{dBc} / \mathrm{Hz}$, corresponding,

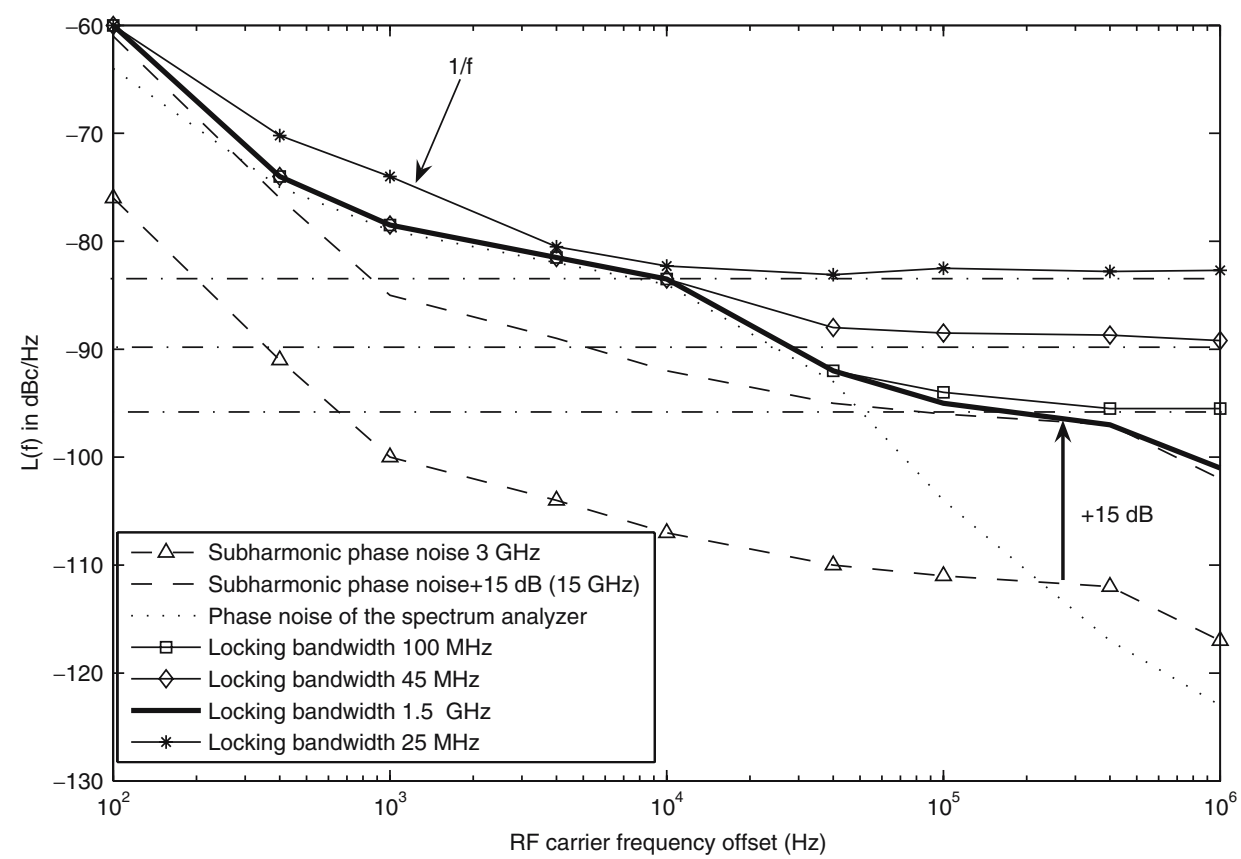

Fig. 3. Phase noise spectrum measurement for different locking bandwidth. 
according to Equation (28), to a path delay mismatch of twice $10 \mathrm{~cm}$ in the experimental setting.

\section{Conclusion}

In this paper, we have established the analytical expression of the phase noise power spectral density of a RF signal generated by the beat note of two lasers locked on two optical modulation side-bands. This analysis includes simultaneously the source oscillator phase noise, the remaining optical phase noises, the true delay and dispersion effects of transmission. We have given numerical results for various parameters of the system, and finally we have experimentally validated the theoretical formulas, which are useful to determine specifications required for efficient modulation formats.

\section{References}

Agrawal, G.P. and R. Roy. Phy. Rev. A 37 2495, 1988.

Bibey, M.-B. et al. IEEE Trans. Microw. Theory Tech. 47 2257, 1999.

Braun, R.P. and G. Grosskopf. 24th European Conference on Optical Communications, 20-24 September 1998. 1665.

Campuzano, G., P. Gallion and G. Debarge. 14th Annual Meeting of the IEEE LEOS, 800, 2001.

Favre, F. IEEE J. Quantum Electron. 23 81, 1987.

Goldberg, L., H.F. Taylor, J.F. Weller and D.M. Bloom. Electron. Lett. 19 491,1983.

Henry, C. H. IEEE J. Quantum Electron. 19 1391, 1983.

Lang, R. IEEE J. Quantum Electron. 18 976, 1982.

Lidoyne, O., P.B. Gallion and D. Erasme. IEEE J. Quantum Electron. 27 344, 1991.

Logan, R.T. IEEE MTT-S Dig. 1741, 1994.

Petermann, K. Laser Diode Modulation and Noise, Kluwer Acadamic Publishers Dordrecht, 123, 1988.

Sayyah, K. and D. Yap. International Topical Meetings on Microwave Photonics, MWP 2003 Proceedings 38 329, 2003.

Schmuck, H., R. Heidemann and R. Hofstetter. Electron. Lett 30, 1994.

Yamamoto, S.N. et al. J. Light Tech. 8 1716, 1990. 